ACUTE

CORONARY

SYNDROMES

\section{The year in cardiology: acute coronary syndromes The year in cardiology 2019}

"Department of Cardiology, John Radcliffe Hospital and University of Oxford, Oxford, United Kingdom

"Fondazione Policlinico Univeristario A. Gemelli, Università Cattolica del Sacro Cuore, Rome, Italy

tRoyal Brompton \& Harefield Hospital, Imperial College, London, United Kingdom

Address for correspondence:

Prof Adrian P. Banning

Department of Cardiology

John Radcliffe Hospital and University of Oxford

Headley Way

Headington

Oxford OX3 9DU

United Kingdom

Email:

profbanning@thecardiologist.co.uk

\section{INTRODUCTION}

The management of acute coronary syndromes (ACS) has made enormous progress over the last 5 decades due to the introduction of defibrillation, beta blockers, thrombolytics, aspirin, primary percutaneous transluminal intervention (PCI), P2Y I 2 inhibitors, statins, radial access, and eventually PCSK9 inhibitors, among others. (I) However, in spite of all these remedies, there is a remaining acute mortality risk, in particular, in those presenting in cardiogenic shock or after resuscitation and an accruing number of major cardiovascular events (MACE) over the following years. ${ }^{(2)}$ Thus, there is an unmet need in the management of ACS. In 2019, there were a number of important papers published in the European Heart Journal and other journals that deepened our knowledge of the spectrum of ACS and their management. Today, patients presenting with acute chest pain and changes in the electrocardiogram (ECG) or biomarkers may have ST-segment elevation myocardial infarction (STEMI) or non-STEMI (NSTEMI) caused by atheroma, coronary dissection, ${ }^{(3)}$ takotsubo syndrome, ${ }^{(4,5)}$ MINOCA (Myocardial infarction with Non Obstructed Coronary Arteries), ${ }^{(6)}$ or myocarditis. ${ }^{(7)}$

\section{GENETICS}

Whole-genome sequencing and early acute myocardial infarction

The relative prevalence and importance of monogenic mutations related to familial hyper-cholesterolaemia $(\mathrm{FH})$ and of high polygenic score (cumulative impact of many common variants) pathways for early-onset myocardial infarction (MI) remain uncertain. Whole-genome sequencing enables simultaneous ascertainment of both monogenic mutations and polygenic score for each individual. Khera, et al. ${ }^{(8)}$ performed whole genome sequencing in 2081 patients hospitalised for earlyonset AMI to assess the prevalence and clinical importance of $\mathrm{FH}$ mutations and a high polygenic score. They observed an $\mathrm{FH}$ mutation in $1.7 \%$ of patients and a high polygenic score in $17 \%$ of patients - each of which was associated with a greater than three-fold increased odds of early-onset AMI. Beyond clinical risk stratification, the polygenic score may additionally foster insights into the mechanistic underpinnings of AMI. Indeed, this risk associated with a high polygenic score is not the result of a discrete underlying mechanism, but rather a quantitative blend of numerous risk pathways.

\section{PATHOPHYSIOLOGY}

Plaque rupture and healing assessed by optical coherence tomography

The mechanisms and the pathologic substrate of plaque erosion and plaque fissure are different. Indeed, plaques complicated by erosion tend to be matrix-rich, lipid-poor, and usually lack prominent macrophage collections, unlike plaques that rupture, which characteristically have thin fibrous caps, large lipid pools, and abundant foam cells. ${ }^{(9)}$ In a prospective study in 211 patients with STEMI who underwent pre-intervention optical coherence tomography (OCT) examination for the culprit lesion, Tan, et al. ${ }^{(10)}$ found that trimethylamine $\mathrm{N}$-oxide (TMAO) levels, a gut microbiota-dependent metabolite derived from dietary phosphatidylcholine and choline, were significantly and independently higher in patients with plaque fissure than in those with plaque erosion. The area under the receiver operating characteristic curve for distinguishing plaque rupture from plaque erosion was 0.89. Thus, plasma TMAO has the potential to serve as a novel biomarker for plaque rupture in 
patients with STEMI, and, indeed, is a prognostic marker in these patients (see below). This might be relevant because risk stratification and management are probably different for plaque fissure and erosion.

Healed plaque ruptures or erosions may be considered as a signature of an aborted ACS. However, the role of plaque healing in the natural history of ischaemic heart disease (IHD) is largely unknown. OCT has been validated for the detection of healed coronary plaques against histology and, therefore, offers the opportunity of assessing their clinical relevance. Vergallo, et al.(II) assessed plaque healing in 2 groups of patients at the extremes of the clinical presentations of IHD: (i) patients with recurring ACS, defined as history of at least 3 AMls or at least 4 ACS with at least I AMI; and (ii) patients with long-standing chronic coronary syndromes, defined as a minimum 3-year history of stable angina in the absence of previous ACS. In the first group, non-culprit plaques only were assessed. They found that patients with recurrent ACS had a distinct atherosclerotic phenotype compared with those with chronic coronary syndrome and longstanding angina, including a much lower prevalence of healed coronary plaques, suggesting that plaque healing may play a role in leading the natural history of patients with IHD.

In another OCT study, Fracassi, et al. ${ }^{(12)}$ assessed plaque healing in the culprit stenosis, among 376 patients with ACS and found plaque healing in more than one quarter. Such patients more frequently were diabetic or hyperlipidaemic; furthermore, healed plaques frequently showed OCT features of local and systemic inflammation. This suggests that the combination of risk factors and local in addition to systemic inflammation may outweigh the protective mechanism of plaque healing and predispose those plaques to develop occlusive thrombus. Thus, a better knowledge of the mechanisms promoting plaque healing might provide new therapeutic targets to reduce ACS burden - in addition to optimal risk factor control.

\section{Mechanisms of coronary microvascular obstruction}

The rapid re-opening and stenting of occluded epicardial coronary arteries via emergency $\mathrm{PCl}$ have revolutionised STEMI treatment. Despite technical refinements and the introduction of numerous antiplatelet and anticoagulant drugs, more than one-third of patients demonstrate coronary microvascular obstruction (CMVO), which deny the benefit of an apparently successful PCl. The mechanisms of CMVO are still largely unknown, while its prevention and treatment remain an unmet need.

Herring, et al.(13) found that STEMI patients with the highest neuropeptide $Y$ levels in the coronary sinus had significantly lower coronary flow reserve, and higher index of microvascular resistance measured with a coronary flow wire, both markers of CMVO. After 2 days, they also had significantly higher levels of myocardial oedema and microvascular obstruction (MVO) on magnetic resonance imaging (MRI), and significantly lower ejection fractions and ventricular dilatation 6 months later. Interestingly, neuropeptide Y (NPY) (100 - 250nM) caused significant vasoconstriction of rat microvascular coronary arteries via increasing vascular smooth muscle calcium waves, and increased coronary vascular resistance and infarct size in Langendorff hearts. These effects were blocked by the $\mathrm{YI}$ receptor antagonist $\mathrm{BIBO} 3304$. Immunohistochemistry of the human coronary microvasculature confirmed the presence of vascular smooth muscle $Y$ I receptors. Thus, antagonism NPY might be an attractive future therapeutic target in the prevention of CMVO (Figure I).

New insight into post-myocardial infarction remodelling The immune response to AMI involves two equally important, consecutive phases: the inflammatory phase and the reparatory phase. During the inflammatory phase, neutrophils and inflammatory $L y 6 C^{\text {hi }}$ monocytes are recruited into the ischaemic myocardium. Subsequently, the Ly $6 \mathrm{C}^{\text {hi }}$ monocytes give rise to reparatory Ly6Clo macrophages, with an important role in cardiac recovery. A balance between the 2 phases is crucial for recovery of cardiac function and patient prognosis. An excessive inflammatory response to AMI amplifies myocardial injury, leading to larger infarcts and loss of function. However, clinical trials testing anti-inflammatory strategies in AMI have so far led to non-significant or even deleterious effects. Ideally, an efficient therapy should inhibit the damaging effects of excessive inflammation, while leaving the repair mechanisms intact.

Alarmins are a group of heterogeneous molecules released from dying cells and activated leucocytes that signal tissue damage and trigger an innate immune response. SIO0A9 and its dimerisation partner SI00A8, also called myeloid-related proteins 8 and 14, are proinflammatory alarmins that are readily produced and stored in large amounts in neutrophils and are increased at the site of acute coronary occlusion. (14) Marinković, et al. ${ }^{(15)}$ studied 524 patients with ACS and found that high plasma SI00A8/A9 at the time of ACS was associated with lower left ventricular ejection fraction (LVEF) at I year and increased hospitalisation for heart failure during follow-up. Moreover, in wild-type C57BL/6 mice with AMI induced by permanent coronary artery ligation, treatment with the SIO0A9 blocker ABR-23890I during the inflammatory phase of the immune response inhibited haematopoietic stem cell proliferation and myeloid cell egression from the bone marrow. The treatment reduced the numbers of neutrophils and monocytes/macrophages in the myocardium, promoted an anti-inflammatory environment, and significantly improved 


\section{Neuropeptide $Y$ and reperfusion post STEMI}

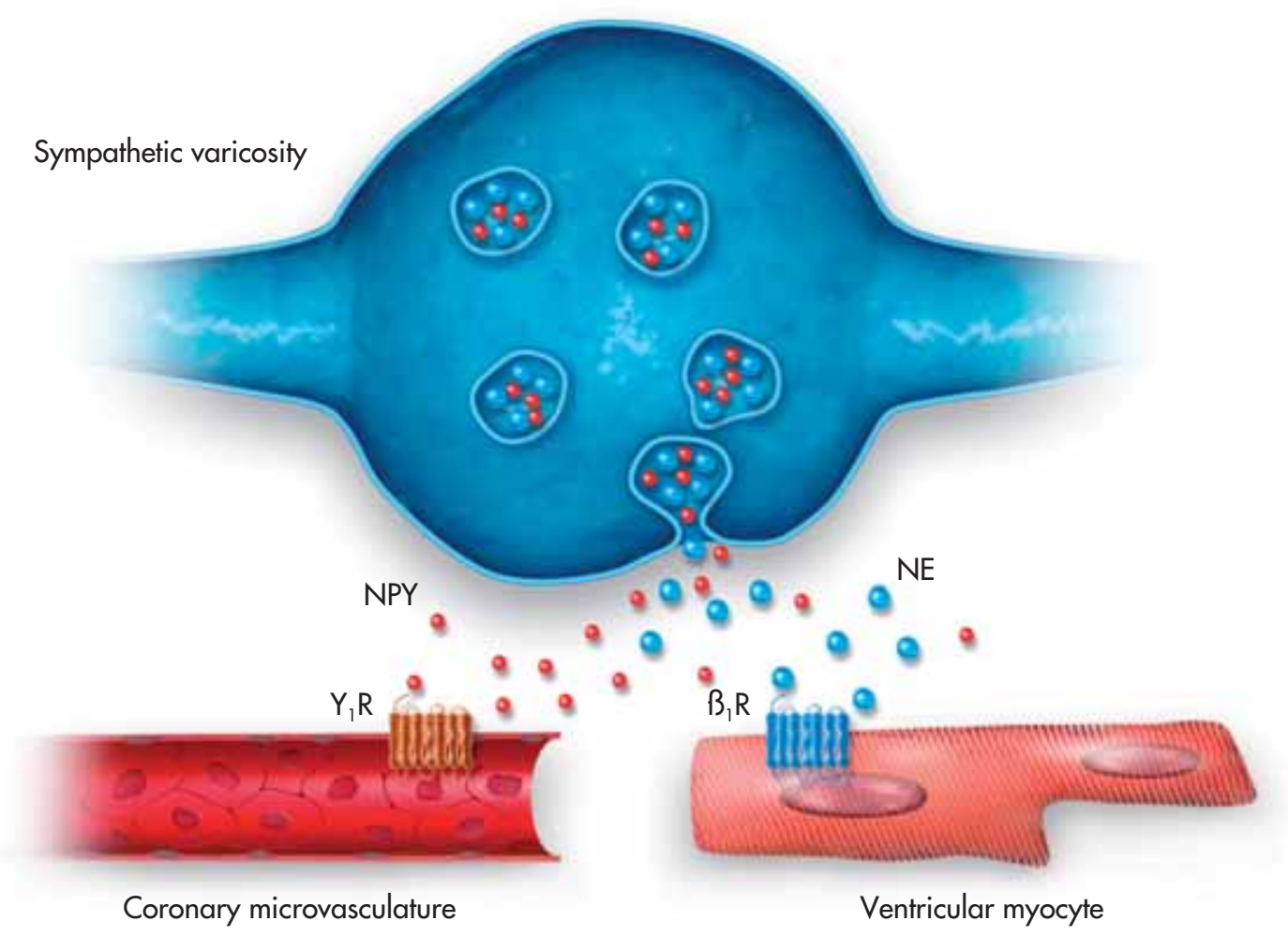

A. Reflow post PPCI

High IMR

Low CFR
B. Microvascular function

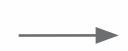

Microvascular obstruction

and more oedema
C. Myocardial infarction

Lower ejection fraction and ventricular dilatation

FIGURE I: Neuropeptide-Y following primary percutaneous coronary intervention for ST-elevation myocardial infarction causes vasoconstriction of the coronary microvasculature and is associated with a high index of microcirculatory resistance and low coronary flow reserve, leading to microvascular obstruction, oedema, and eventually a lower ejection fraction and ventricular dilatation. Reproduced with permission from ref. ${ }^{(13)}$

cardiac function compared with controls. To mimic the clinical scenario, they further confirmed the effects of the treatment in a mouse model of ischaemia and reperfusion. Compared with untreated mice, 3-day ABR-23890I treatment significantly improved LVEF. Thus, SIO0A9 blockade might represent a feasible strategy to improve prognosis in ACS patients.

Tang, et al. ${ }^{(16)}$ investigated the effects of gut microbiota on cardiac repair after AMI. C57BL/6) mice were treated with antibiotics 7 days before AMI to deplete mouse gut microbiota. Antibiotic-treated mice displayed drastic, dose-dependent mortality after AMI, associated with a reorganisation of the gut microbial community such as a reduction in Lactobacillus. The physiological status and survival of mice were significantly improved after faecal reconstitution or dietary supplementation with short-chain fatty acids that are altered after antibiotic treatment; these benefits appeared to be mediated by immunomodulatory effects. In addition, supplementing antibiotictreated mice with a Lactobacillus probiotic before AMI restored yielded cardioprotective effects. Thus, this study uncovers the adverse effects of antibiotics on survival after $\mathrm{Ml}$ and addresses a promising therapeutic strategy that involves modulation of gut microbiota composition through probiotic supplementation (Figure 2). 


\section{A}

M Gut microbiota

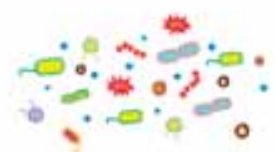

F Short-chain fatty acid

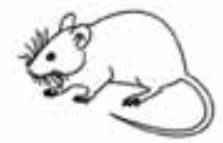

Untreated

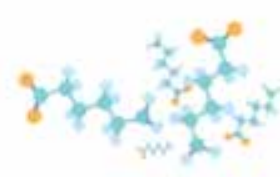

I Myeloid immune

composition

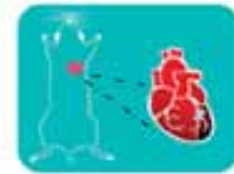

Normal survival

MI

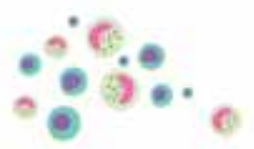

B
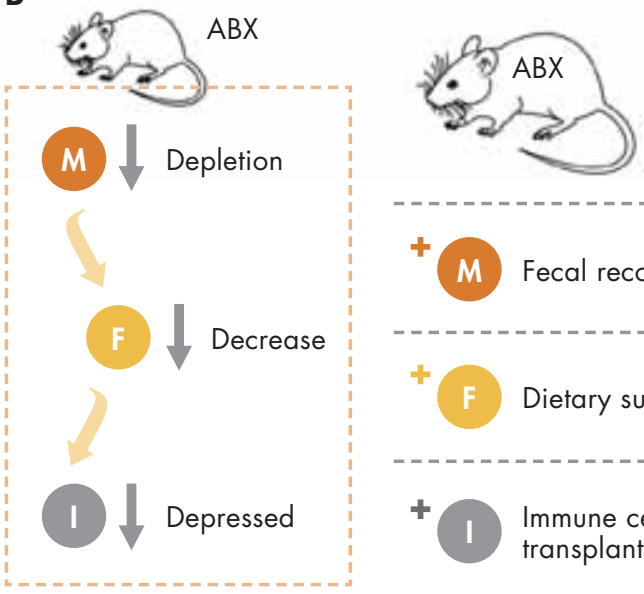

MI
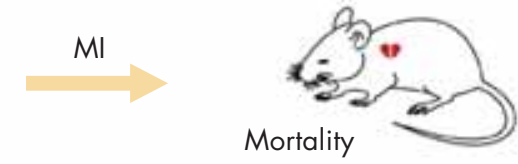

M Fecal reconstitution
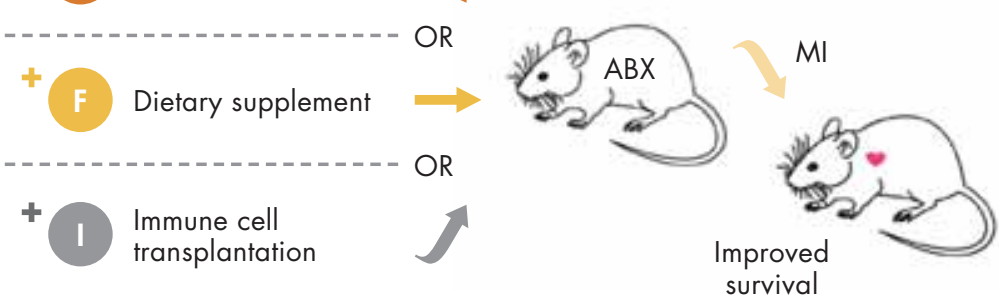

M Gut microbiota

F Short-chain fatty acid
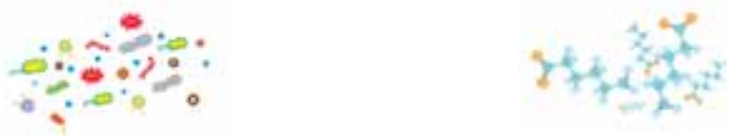

I Myeloid immune composition

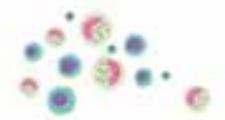

FIGURE 2: A schaematic diagram showing the importance of gut microbiota-derived short-chain fatty acids for maintaining immune composition and repair capacity during homoeostasis.

(A) In untreated mice, gut microbiota-derived short-chain fatty acids modulate immune composition and repair capacity during homoeostasis. (B) On antibiotics-mediated dysbiosis, the depletion of the gut microbiota and the short-chain fatty acid products are associated with changes in immune composition and an abolishment of repair response, leading to exacerbated mortality after myocardial infarction. Restoration of the system across each level through the faecal reconstitution of the gut microbiota, dietary supplementation of short-chain fatty acids, or immune cell transplantation results in improved survival. Reproduced with permission from ref.(16)

\section{Mechanisms of takotsubo syndrome}

In 55 patients with takotsubo syndrome, Scally, et al.(17) found myocardial macrophage inflammatory infiltrates as assessed by MRI as well as changes in the distribution of monocyte subsets and an increase in systemic pro-inflammatory cytokines. Many of these changes persisted for at least 5 months, suggesting a low-grade chronic inflammatory state. Obviously, whether inflammation is a cause or a consequence of the acute takotsubo event remains to be shown. Moreover, whether inflammation is maladaptive and implicated in the persistence in the 
long-term consequences of this condition is uncertain. Nevertheless, inflammation might play a role in the complex pathogenesis of this syndrome.

Most importantly, novel investigations using functional MRI of the brain suggest that an altered limbic and central autonomic signal processing plays a crucial role in takotsubo and may explain the inappropriately excessive reaction of the sympathetic nervous system to stressful triggers. Thus, takotsubo is indeed a brain disease and the heart may just represent the target organ. ${ }^{(18)}$

\section{DIAGNOSIS}

\section{Troponins}

Boeddinghaus, et al. ${ }^{(19)}$ prospectively enrolled patients presenting with symptoms suggestive of AMI in 3 large diagnostic studies in order to assess the validity of the $0 / \mathrm{h}$-algorithms according to age ( $<55$ years, $\geq 55-<70$ years, and $\geq 70$ years). Rule-out safety of the ESC high-sensitivity cardiac troponin $T$ (hs-cTnT) 0/I h-algorithm was very high with a sensitivity of $\mathbf{9 9 . 3 \%}$ in all age strata, while triage efficacy decreased with increasing age with sensitivity dropping from 93\% - 55\%. Slightly higher cut-off concentrations optimised for older patients maintained very high safety of rule-out and increased specificity. Findings were confirmed in 2 validation cohorts and also for hsTnl. While safety of the ESC $0 /$ I h-algorithms remained very high, increasing age significantly reduced overall efficacy and the accuracy of rule-in. Thus, alternative slightly higher cut-off concentrations may be considered for older patients, although the problem remains for other confounders like chronic kidney disease, chronic heart failure, atrial fibrillation, and others that also need to be incorporated. Twerenbold, et al.(20) confirmed the excellent applicability, short time to emergency department discharge, and low rate of 30-day MACE associated with the routine clinical use of the ESC $0 / 1-h$-algorithm for the management of patients presenting with acute chest discomfort to the emergency department in a real-world setting.

Finally, important concepts for institutional transition to hs-cTn methodology providing recommendations useful for education before implementation have been reported in an Expert Panel published by Januzzi, et al.(21)

\section{Implantable cardiac alert system}

Symptoms remain a poor prompt for ACS. In a multicentre, randomised trial of an implantable cardiac monitor that included 907 high-risk ACS patients with rapidly progressive ST-segment deviation randomised to a control (alarms deactivated) or treatment group for 6 months, after which alarms were activated in all subjects, ${ }^{(22)}$ safety revealed a 96.7\% freedom from system-related complications. The pri- mary efficacy endpoint of cardiac or unexplained death, new Q-wave $\mathrm{MI}$, and detection to presentation time $>2$ hours following a confirmed occlusive event within 7 days was numerically, but not statistically, reduced among patients with activated alarms. When the observation window was extended to 50, 70, and 90 days in a pre-specified analysis to include the majority of confirmed occlusive events in the control group, and an exploratory dual-baseline ECG analysis was used to reduce noise, a significant reduction in the primary endpoint was observed. Moreover, alarms significantly decreased detection to arrival time at a medical facility. This device may be beneficial among high-risk subjects in potentially identifying asymptomatic events.

\section{RISK STRATIFICATION}

\section{Biomarkers}

Among 4257 patients of the VISTA-16 trial, initial and subsequent increases in high-sensitivity C-reactive protein (hsCRP) levels during 16 weeks after ACS were associated with a greater risk of the combined MACE endpoint, cardiovascular (CV) death, and all-cause death - despite established background therapies. ${ }^{(23)}$ Further studies will be required to determine whether initial and serial hsCRP measurements can help guide the use of targeted anti-inflammatory therapies after ACS or whether more specific inflammatory markers will be needed to this end.

Four new biomarkers have been investigated in different trials in the setting of ACS: (i) galectin-3, implicated in fibrosis; ${ }^{(24)}$ (ii) impaired endogenous fibrinolysis;, (25) (iii) trimethyllysine and TMAO, gut-microbiota derived metabolite; ${ }^{(26)}$ and (iv) serum cholesterol efflux capacity. (27) All these biomarkers were found to be independently associated with MACE at follow-up. Again, further studies will be required to establish whether these markers identify patient subsets who need personalised forms of treatment.

Finally, Lindholm, et al.(28) assessed the association between cystatin-C, growth differentiation factor-I5 (GDF-15), hsCRP, hs-TnT and Tnl, and N-terminal pro-B-type natriuretic peptide (NT-proBNP) and specific causes of mortality among 17095 ACS patients of the PLATO trial. They found that NTproBNP and GDF-I5 were strong markers associated with all-cause death based on their associations with death due to heart failure as well as due to arrhythmia and sudden cardiac death. Growth differentiation factor-15 had the strongest associations with death due to other vascular or non-vascular causes and possibly with death due to bleeding. It remains to be established how this important prognostic information can guide treatment. 


\section{Cardiac magnetic resonance and entropy}

Entropy is a new late gadolinium-enhanced MRI-derived parameter to evaluate tissue inhomogeneity, independent of signal intensity thresholds. Androulakis, et al. ${ }^{(29)}$ enrolled I 54 consecutive post-AMI patients undergoing late gadolinium-enhanced MRI prior to implantable cardioverter-defibrillator (ICD) implantation. When entropy involved the entire left ventricle $(\mathrm{LV})$, this was associated with mortality. After adjusting for multivessel disease, acute revascularisation, and ejection fraction, entropy of the scar was independently associated with the presence of ventricular arrhythmias. The association between LV entropy and mortality may reflect adverse and irreversible, inhomogeneous remodelling of the post-infarct LV and/or possibly fatal arrhythmias. Further studies are warranted to establish whether this new marker can allow a better identification of candidates for ICD after AMI.

\section{TREATMENT}

Ticagrelor compared to prasugrel were studied in the ISARREACT 5 study (Figure 3). ${ }^{(30)}$ Patients with ACS and a proposed interventional strategy were randomised and the pri-

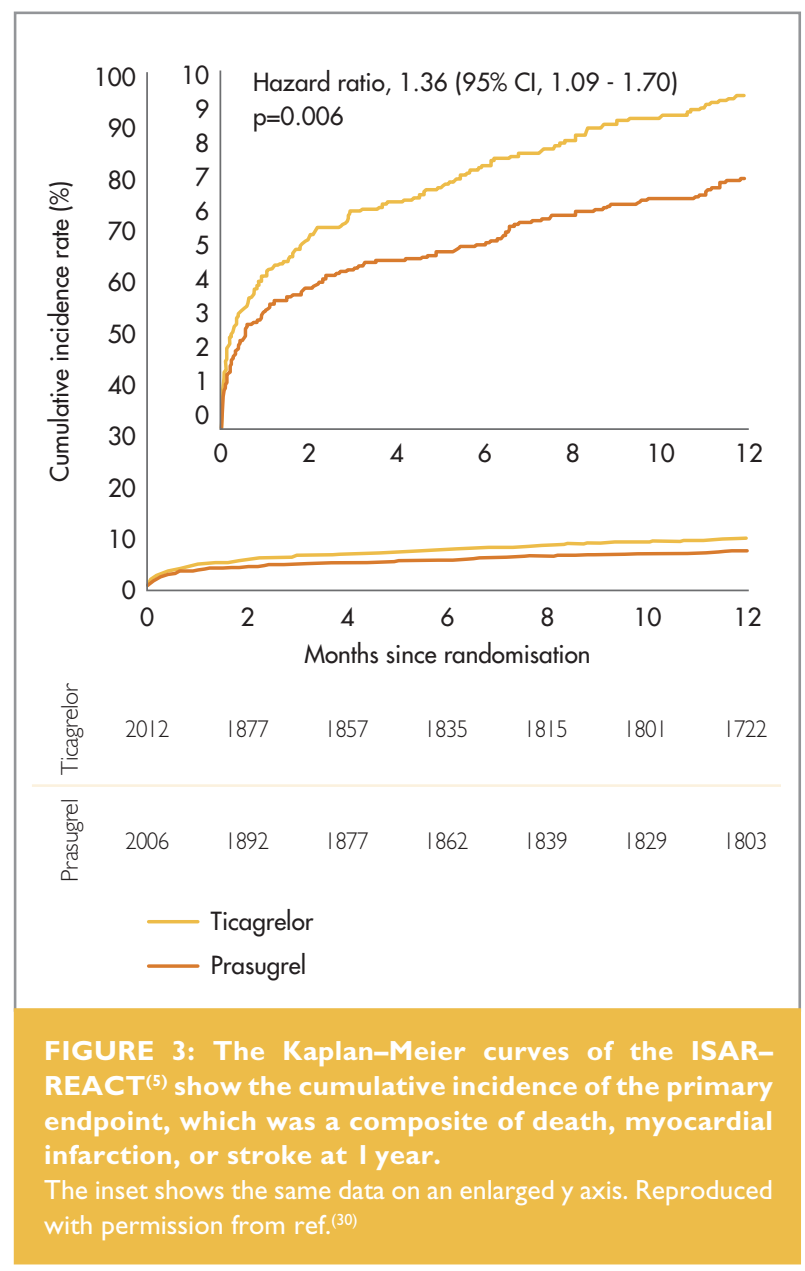

mary composite endpoint was death, Ml, or stroke. In these patients with or without STEMI, prasugrel therapy was superior to ticagrelor with no difference in bleeding. These important comparative data are likely to change clinical practice.

\section{Timing of treatment}

The optimal timing of administration of dual antiplatelet therapy (DAPT) in STEMI patients was investigated by the Swedish Coronary Angiography and Angioplasty Registry. ${ }^{(3)}$ Patients were stratified between either post- or pre-procedure treatment with P2Y 12 receptor antagonists. Of the 44804 patients, $58 \%$ had been on clopidogrel, $35 \%$ on ticagrelor, and $5 \%$ on prasugrel. There was no survival benefit from pre-treatment or any impact on infarct-related artery patency, stent thrombosis, or bleeding. These data are surprising as a potential impact on early stent thrombosis, in particular, might have been anticipated. Existing Guidelines(32) recommend catheter-lab administration of antiplatelet drugs and these data are supportive of that strategy, although Abtan and Steg ${ }^{(33)}$ argue that we should continue to give $\mathrm{P} 2 \mathrm{Y} 12$ receptor antagonists as early as possible in probable STEMI patients, as there is a strong biological plausibility of likely benefit with no obvious likely detriment.

Whether patients with transient ST-elevation (patients who present initially with ST-elevation on the electrocardiogram but, subsequently, show complete normalisation of the STsegment and relief of symptoms before reperfusion therapy) require immediate revascularisation was studied in a trial ${ }^{(34)}$ of 142 patients who were randomised to immediate (mean 0.3 hours) or delayed angiography (mean 22.7 hours). The outcome was infarct size by MRI at Day 4. The observed infarcts were generally small and there was no clear benefit from the immediate strategy, although 4/7I patients required urgent intervention because of further symptoms and ST elevation while waiting. Consequently, these data can probably be interpreted as allowing decisions about timing of revascularisation in patients with transient STEMI, to be guided by the availability of angiography and careful clinical assessment.

The management of non-culprit lesions in STEMI patients has been extensively discussed over the last years. Small randomised trials have suggested a probable benefit from complete revascularisation. The large COMPLETE trial randomised 4 04I STEMI patients (Figure 4) to complete or partial revascularisation. ${ }^{(35)}$ Complete revascularisation was superior to culprit-only as it reduced the risk of CV death or AMI. There was also benefit from complete revascularisation in reducing ischaemic endpoints. Timing appeared to be less important and deferring any secondary revascularisation procedure was safe. Thus, the advantage of complete revascularisation will presumably be reflected in subsequent ESC Guidelines. 


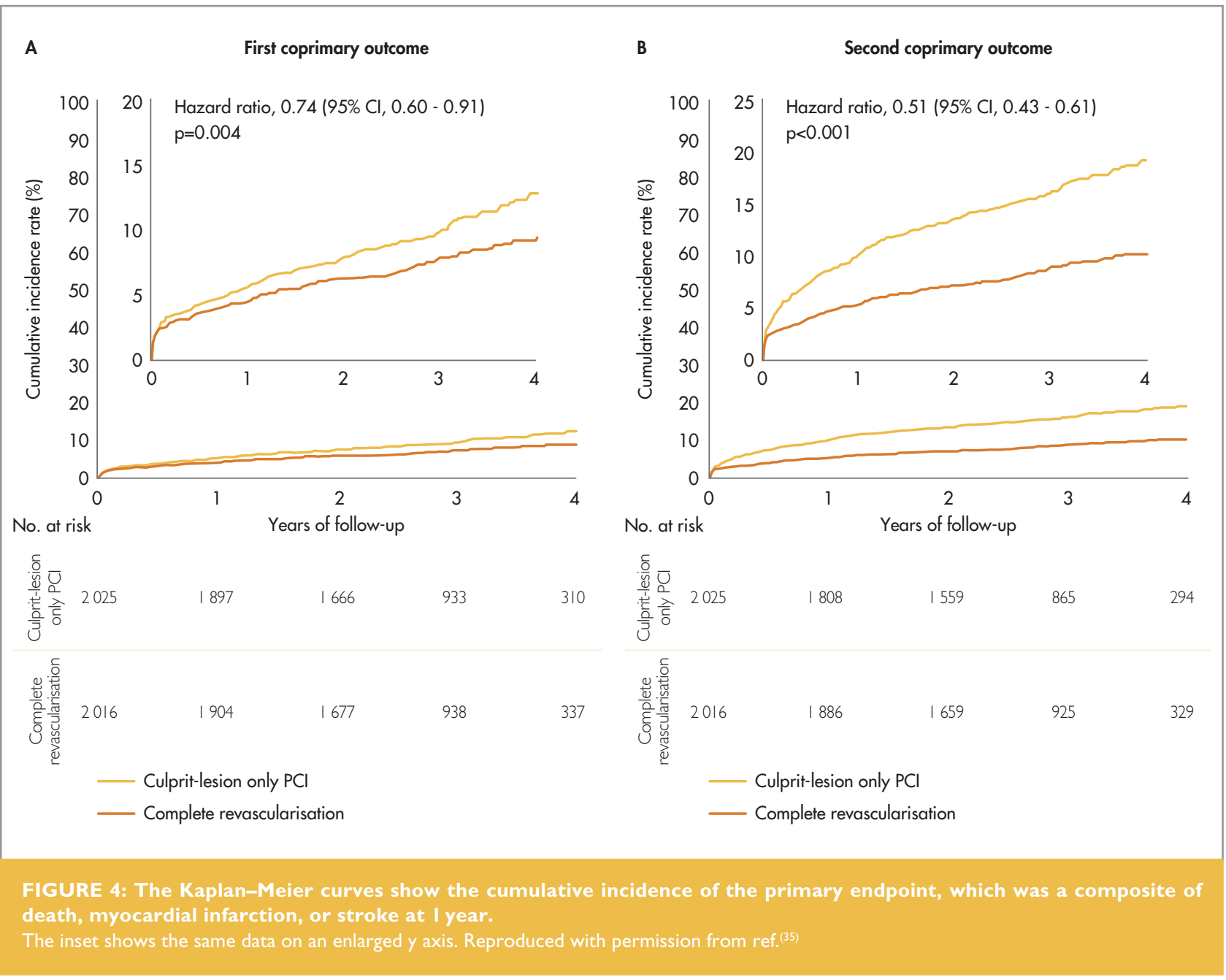

Can we tailor treatment in acute coronary syndromes? There remains a desire to tailor drug therapy to the individual patients and perhaps the individual lesion. This strategy would allow pharmaceutical optimisation of protection with minimisation of side effects or bleeding risk. The CHAMPION PHOENIX trial(36) has suggested that complex coronary lesions can be characterised and that it is inadequate treatment of these "high risk" lesions that leads to repeat revascularisation or clinical events. Compared with a loading dose of clopidogrel, cangrelor reduced MACE occurring within 48 hours after $\mathrm{PCl}$ in patients with ACS, regardless of baseline lesion complexity. However, the absolute benefit: risk profile for cangrelor was greatest during $\mathrm{PCl}$ in complex coronary anatomy. In a substudy of the PROSPECT trial,(37) virtual histology and grey scale intravascular ultrasound (IVUS) was a predictor of a suboptimal final angiographic result reflected as a high residual Syntax Score after intervention. A detailed Expert consensus review has provided detail on characterisation of acute coronary lesions using IVUS or OCT and suggested a triaged therapeutic approach. ${ }^{(38)}$
Unfortunately measuring individuals' responses to antiplatelet drugs has not shown clinical utility. Randomising patients to measuring platelet function in the TROPICAL trial(39) showed no measurable clinical advantage. Within the trial, those patients with maximal platelet inhibition were predictably most prone to bleeding.

Optimising treatment in ST-segment elevation myocardial infarction

About one-third of STEMI patients have suboptimal reperfusion after $\mathrm{PCl}$ and it is this group of patients that might experience heart failure and premature death. MRI can provide evidence of MVO and myocardial haemorrhage. ${ }^{(40)}$ In the DANAMI-3 trial, delayed presentation ${ }^{(41)}$ as evidenced by prolonged door-to-wire-time predicted adverse clinical outcome, consistent with previous observations using intracoronary physiology measurements suggesting increased risk in these "late presenters". (42) Enhanced and continued public information campaigns are required to optimise outcomes in high-risk STEMI patients along with novel adjunctive therapies. In this 
context, full dose intracoronary tenectaplase was inferior to abciximab in a small randomised trial(43) and using low dose intracoronary tenecteplase after reperfusion in the T-time trial also failed to reduce MVO measured using cardiac magnetic resonance imaging (CMRI). ${ }^{(44)}$

Hypothesis generating results were presented by the Microvascular Reperfusion Utilising Sonothrombolysis in Acute Myocardial Infarction (MRUSMI) investigators. ${ }^{(45)}$ They randomised STEMI patients to either standard $\mathrm{PCl}$ or diagnostic ultrasound transducer guided, high mechanical index impulses during an ultrasound agent transfusion prior to and following $\mathrm{PCl}$. The ultrasound's high mechanical index impulses create microbubble cavitation that induce shear forces, designed to dissolve intracoronary thrombi. The treatment cohort demonstrated higher recanalisation (48\% vs. 20\%) and TIMI 3 flow rates (32\% vs. $14 \%$ ) within the infarcted vessel and ST-segment resolution prior to primary $\mathrm{PCl}$ occurred more frequently. Furthermore, infarct size as assessed by MRI and TNT peak values was also reduced by the intervention. Optimising reperfusion for patients with STEMI when there is lot of thrombus is challenging and these preliminary results hint at a possible alternate approach.

Remote preconditioning in STEMI was studied in the CONDI-2/ ERIC PPCI trial, ${ }^{(46)}$ which randomised 540 I patients to standard treatment (including a sham simulated remote ischaemic conditioning at UK sites) or remote ischaemic conditioning (intermittent ischaemia and reperfusion applied to the arm through 4 cycles of 5 minutes inflation and 5 minutes deflation of an automated cuff device) before primary PCl. Unfortunately, remote ischaemic conditioning did not improve clinical outcomes (cardiac death or hospitalisation for heart failure) at 12 months. New approaches and therapies within this area are warranted if this experimentally well-documented concept should ever reach the clinical applicability.

\section{OUTCOMES}

Quality of treatment and outcomes

In a cohort of 389507 NSTEMI patients, optimal care, defined as the receipt of all eligible treatments, was inversely related to risk status (defined by the GRACE risk score), i.e. $25.6 \%$ in low, $18.6 \%$ in intermediate, and $11.5 \%$ in high-risk patients. At the end of 2.3 years of follow-up, the association between the use of all eligible guideline-indicated treatments and improved survival remained only significant for high-risk NSTEMI. ${ }^{(47)}$ Thus, optimal use of guideline-indicated care for NSTEMI was associated with greater survival gains with increasing GRACE risk, but its use paradoxically decreased with increasing GRACE risk - thus leaving room for improvement.

In the SWEDHEART registry, outcomes of patients presenting with NSTEMI followed over 20 years demonstrated a substantial improvement in long-term survival and reduction in the risk of MACE.(48) These improvements were associated with the gradual uptake and widespread use of $\mathrm{PCl}$ and longterm use of evidence-based medications, consistent with the anticipated effectiveness of their implementation as proposed in guidelines (Figure 5).

The value of a cardiac rehabilitation programme was compared in 839 patients who attended a planned programme at discharge after ACS or surgical revascularisation, while 44I patients were discharged without it. ${ }^{(49)}$ At multivariable Cox proportional hazard analysis, the cardiac rehabilitation programme was an independent predictor of lower occurrence of MACE (hazard ratio 0.55), while in a propensity-matched analysis patients attending the cardiac rehabilitation programme also experienced a lower total mortality (10\% vs. 19\%) and CV mortality (2\% vs. $7 \%$ ) compared to non-rehabilitated ones. Thus, the positive effects of ambulatory cardiac rehabilitation are also notable in a real-world population.

Finally, in a cohort study of 123780 consecutive PCls from the Pan-London PCl Registry, the outcomes pre- and post-public reporting in the UK were compared. ${ }^{(50)}$ After public reporting was introduced, patients were older and had more complex medical problems, while in-hospital MACE and MACCE and 30-day mortality rates were significantly lower. These results probably reflect continued improvements in $\mathrm{PCl}$ outcomes concomitant with the introduction of public reporting, but the lower reported complication rate could also reflect a change in the documentation of risk factors and a change in operator behaviour. Reassuring, additional data from the UK registry also confirm continued temporal improvement in outcomes in ACS and notably demonstrate no difference in outcome depending on the times of day that treatment occurred. Patients treated as emergencies at night had similar outcomes to those treated within "office hours".

\section{Modes of death after non-ST-segment elevation myocardial infarction}

In 66252 patients with NSTEMI enrolled in 14 TIMI trials, baseline characteristics and modes and timing of death were examined. Of the 2606 patients with known modes of death, $75.1 \%$ were related to MACE, 3.0\% were related to a bleeding event (including intracranial haemorrhage), and $21.8 \%$ were 


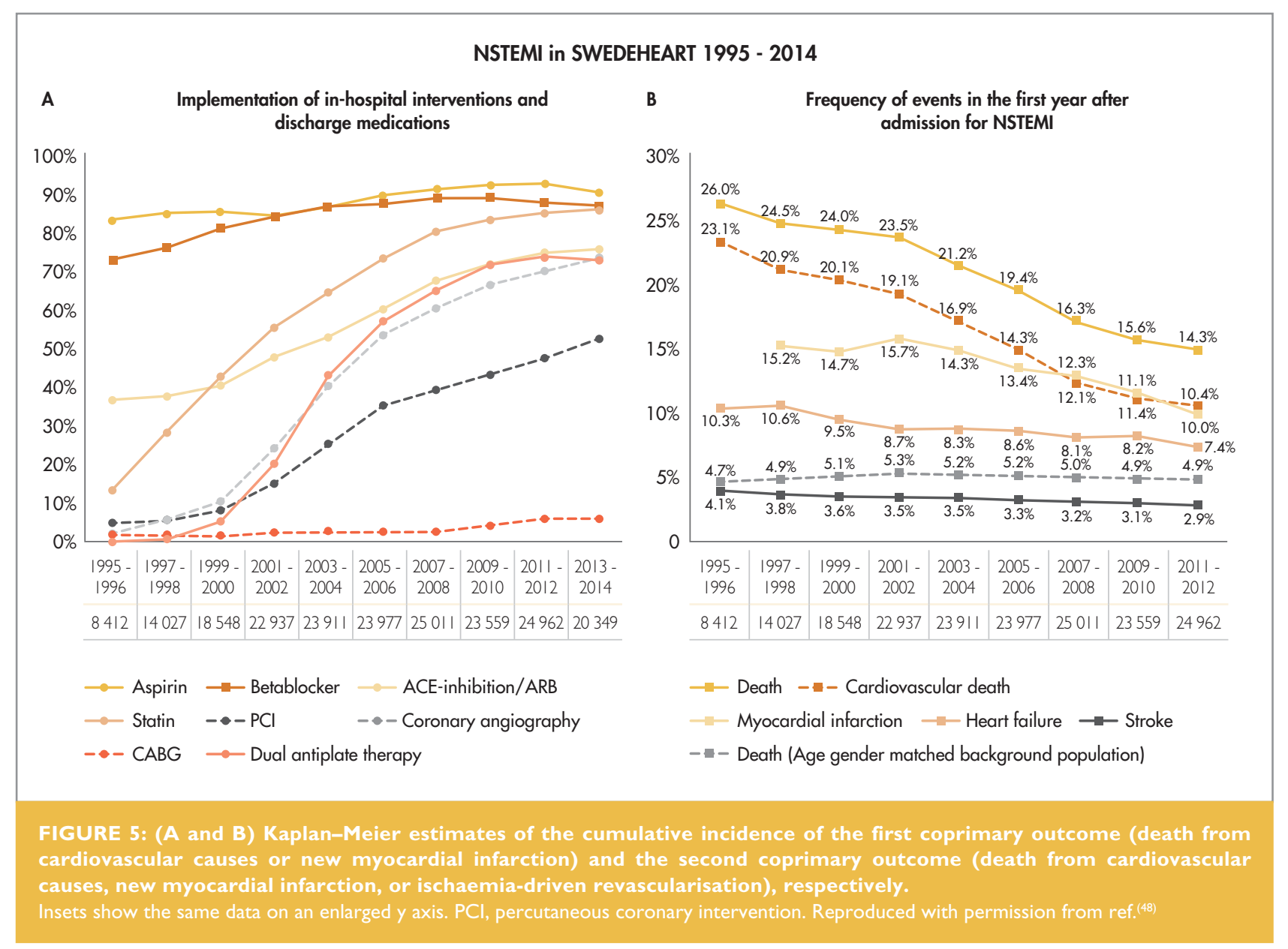

related to a non-CV/non-bleeding event. ${ }^{(51)}$ The most common modes of $\mathrm{CV}$ death were sudden death and recurrent AMI (36.4\% and 23.4\%, respectively). The proportion of CV deaths related to recurrent $\mathrm{AMI}$ was higher in the first 30 days than it was after 30 days following NSTEMI (30.6\% vs. $18.7 \%)$, whereas the proportion of sudden death was lower in the first 30 days than after 30 days (21.6\% vs. 46.2\%). Thus, sudden death represented the largest proportion of CV deaths after 30 days. Further investigations aimed at defining management approaches to reduce sudden death following NSTEMI may be critical to reducing late mortality.

\section{Outcomes of in-hospital acute coronary syndromes}

In a cohort study of 1.3 million patients hospitalised in US Veterans Health Administration facilities, an incidence of inhospital AMI of 4.27 per I 000 admissions and risk factors associated with in-hospital AMI such as history of IHD, elevated heart rate, low haemoglobin level, and elevated white blood cell count were reported. ${ }^{(52)}$ Compared with matched controls, mortality was significantly higher for in-hospital AMI. Thus, in-hospital AMI is common and associated with com- mon CV risk factors and markers of acute illness and with high mortality approaching $60 \%$ at 1 year. Further studies of in-hospital AMI may yield opportunities to reduce in-hospital AMI risk and improve patient outcomes.

\section{Outcomes of atypical acute coronary syndromes}

\section{Spontaneous coronary artery dissection}

Spontaneous coronary artery dissection (SCAD) is an underdiagnosed and poorly understood condition and an important cause of AMI in young women. ${ }^{(3)}$ In the Canadian multicentre, prospective, observational study of 750 patients, predisposing conditions included fibromuscular dysplasia in $31.1 \%$, systemic inflammatory diseases in $4.7 \%$, peripartum in $4.5 \%$, and connective tissue disorders in $3.6 \%$ were noted. ${ }^{(53)}$ Most were treated conservatively, while $14 \%$ underwent $\mathrm{PCl}$ and a few coronary artery bypass surgery. In-hospital composite MACE was $8.8 \%$, but higher in peripartum SCAD (20.6\% vs. 8.2\%). Overall 30-day MACE was $8.8 \%$ with peripartum SCAD and connective tissue disease being independent predictors of 30-day MACE. 


\section{Takotsubo syndrome}

Two different studies of the InterTak Registry including over I 500 patients with takotsubo syndrome found that both inhospital cardiac arrest (4.9\% of patients) ${ }^{(54)}$ or the presence of an associated malignancy (16.6\% of patients) ${ }^{(55)}$ were associated with worse long-term outcome. Of note, patients with cardiac arrest were more likely to be younger, male, and have apical takotsubo, atrial fibrillation, neurologic comorbidities, physical triggers, longer corrected QT-interval, and lower LVEF, while those with malignancy were older and more likely to have physical triggers, but less likely to have emotional triggers.

\section{Myocardial infarction with non-obstructed}

\section{coronary arteries}

The long-term outcome of MINOCA was investigated in a large US Registry of 286780 AMI admissions, of which 5.9\% had MINOCA.(56) Following risk-adjustment, MINOCA patients had a $43 \%$ lower risk of MACE over 12 months compared to those with AMI and coronary artery disease. This pattern was similar for adjusted risks of the MACE components. Thus, MINOCA has an unfavourable prognosis in elderly patients with 1 in 5 suffering a major adverse event over 12 months.

\section{The Spectrum of ACS}

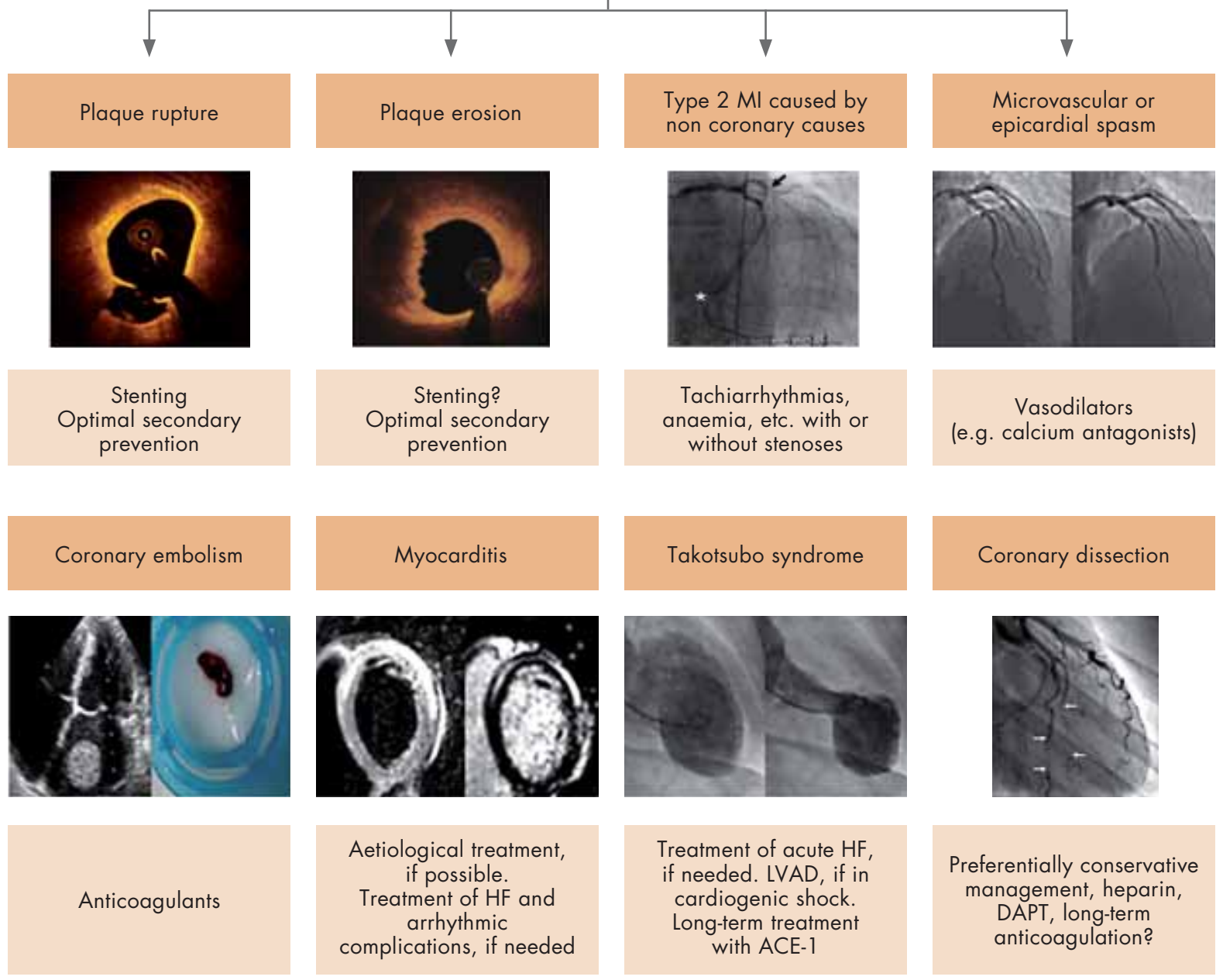

FIGURE 6: The spectrum of acute coronary syndromes encompasses plaque rupture and erosion (= Type I myocardial infarction), Type 2 myocardial infarction, epicardial and microvascular coronary spasm, coronary embolism, acute myocarditis, takotsubo syndrome and coronary dissection. 


\section{Women vs. men}

The impact of gender was assessed in 13451 NSTEMI and STEMI patients undergoing $\mathrm{PCl}$ in the Victorian Cardiac Outcomes Registry in Australia.(57) Women with STEMI had significant delays in presentation and revascularisation with a higher 30-day mortality compared with men, while women with NSTEMI had no delay in presentation or revascularisation, with mortality comparable to men. Thus, public awareness campaigns might be needed to address women's recognition and early action for STEMI.

In a Swiss population of 4360 patients with STEMI, ischaemic time in women remained greater than that in men due to persistently greater patient delays. ${ }^{(58)}$ In contrast to men, clinical signs of ongoing chest discomfort did not predict delays in women, suggesting that female STEMI patients were less likely to attribute symptoms to a condition requiring urgent treatment.

In a Chinese population of 82196 patients, women hospitalised for ACS less frequently received acute treatments and strategies for secondary prevention than men.(59) The observed sex differences in in-hospital mortality were mainly due to worse clinical profiles and fewer evidence-based acute treatments provided to women with ACS.

Finally, Vicent, et al. ${ }^{(60)}$ in an octogenarian Spanish population of 535 patients with STEMI found that female sex was independently associated with death and hospitalisation at 6-month follow-up.

Medicaid beneficiaries vs. privately insured individuals In 42645 and 171 545 STEMls receiving Medicaid or private insurance, respectively, ${ }^{(61)}$ in unadjusted analyses, Medicaid beneficiaries had lower rates of coronary revascularisation and higher rates of in-hospital mortality compared with privately insured individuals. Similar results were found in propensitymatched cohorts. Further studies are needed to identify and understand the causes of the variation in STEMI outcomes by insurance status.

\section{Black vs. White patients}

In a US cohort of 6402 patients, self-identified black and white patients differed in several clinical and socioeconomic characteristics. ${ }^{(62)}$ The higher the prevalence of characteristics associated with being black, the higher the 5-year mortality rate, but no differences were observed between black and white patients with similar characteristics. Thus, a greater prevalence of characteristics associated with black race, but not race itself, was associated with higher mortality risk after AMI.

\section{CONCLUSIONS}

Important data from large randomised trials have augmented the management of patients presenting with ACS. Importantly, recognition of the clinical spectrum of ACS has expanded in recent years and atypical forms, besides the classical STEMI and NSTEMI, such as takotsubo syndrome, coronary dissection, MINOCA, and myocarditis, have been increasingly recognised, characterised and their causes and mechanisms defined (Figure 6). The management of these novel forms of ACS is still not evidence-based, but significant progress has been made recently. Furthermore, disparities in the implementation of guideline-based therapies are increasingly addressed to the benefit of the patient population at large.

The opinions expressed in this article are not necessarily those of the Editors of the European Heart Journal or of the European Society of Cardiology.

\section{ACKNOWLEDGEMENTS}

The authors would wish to thank Susanne Dedecke for her support.

Conflict of interest: A.B. is partially funded by the NHS NIHR Oxford Biomedical Research Centre and reports an Institutional Educational Grant from Boston Scientific and honoraria from Abbott, Boston Scientific, Medtronic, and Miracor. F.C. reports speaker fees by Amgen, BMS, Servier, Menarini, Novartis, and Astra Zeneca, and TFL reports research grant or honoraria by Abbott, Amgen, Biotronik, Boston Scientific, Eli Lilly, Medtronic, and Sanofi-Regeneron. 
I. Luscher TF, Obeid S. From Eisenhower's heart attack to modern management: A true success story! Eur Heart J 20 17;38:3066-3069.

2. Szummer K, Wallentin L, Lindhagen L, et al. Improved outcomes in patients with ST-elevation myocardial infarction during the last 20 years are related to implementation of evidence-based treatments: experiences from the SWEDEHEART registry 1995-20 I 4. Eur Heart J 20 I7:38:3056-3065.

3. Adlam D, Alfonso F, Maas A, et al.; Writing Committee. European Society of Cardiology, acute cardiovascular care association, SCAD study group: A position paper on spontaneous coronary artery dissection. Eur Heart J 2018;39:3353-3368.

4. Ghadri J-R, Wittstein IS, Prasad A, et al. International Expert Consensus Document on Takotsubo Syndrome (Part I): Clinical characteristics, diagnostic criteria, and pathophysiology. Eur Heart J 20 I 8;39:2032-2046.

5. Ghadri J-R, Wittstein IS, Prasad A, et al. International Expert Consensus Document on Takotsubo Syndrome (Part II): Diagnostic workup, outcome, and management. Eur Heart J 2018;39:2047-2062.

6. Niccoli G, Scalone G, Crea F. Acute myocardial infarction with no obstructive coronary atherosclerosis: mechanisms and management. Eur Heart J 2015;36:475-48।.

7. Ekstrom K, Lehtonen J, Nordenswan HK, et al. Sudden death in cardiac sarcoidosis: An analysis of nationwide clinical and cause-of-death registries. Eur Heart J 2019;40:3121-3128.

8. Khera AV, Chaffin M, Zekavat SM, et al. Whole-genome sequencing to characterise monogenic and polygenic contributions in patients hospitalised with early-onset myocardial infarction. Circulation 2019;139:1593-1602.

9. Libby P, Pasterkamp G, Crea F, et al. Reassessing the mechanisms of acute coronary syndromes. Circ Res 2019;124:150-160.

10. Tan Y, Sheng Z, Zhou P, et al. Plasma trimethylamine N-oxide as a novel biomarker for plaque rupture in patients with ST-segment-elevation myocardial infarction. Circ Cardiovasc Interv 2019;12:e007281.

II. Vergallo R, Porto I, D'Amario D, et al. Coronary atherosclerotic phenotype and plaque healing in patients with recurrent acute coronary syndromes compared with patients with long-term clinical stability: An in vivo optical coherence tomography study. JAMA Cardiol 2019;4:321-329.

12. Fracassi F, Crea F, Sugiyama $T$, et al. Healed culprit plaques in patients with acute coronary syndromes. J Am Coll Cardiol 2019;73:2253-2263.

13. Herring N, Tapoulal N, Kalla M, et al.; Oxford Acute Myocardial Infarction (OxAMI) Study. Neuropeptide-Y causes coronary microvascular constriction and is associated with reduced ejection fraction following ST-elevation myocardial infarction. Eur Heart J 2019;40:1920-1929.

14. Altwegg LA, Neidhart M, Hersberger M, et al. Myeloid-related protein 8/14 complex is released by monocytes and granulocytes at the site of coronary occlusion: A novel, early, and sensitive marker of acute coronary syndromes. Eur Heart J 2007;28:941-948.

15. Marinković G, Grauen Larsen H, Yndigegn T, et al. Inhibition of proinflammatory myeloid cell responses by short-term SI00A9 blockade improves cardiac function after myocardial infarction. Eur Heart J 2019; 40:2713-2723.

16. Tang TWH, Chen HC, Chen CY, et al. Loss of gut microbiota alters immune system composition and cripples postinfarction cardiac repair. Circulation 2019;139:647-659.

17. Scally C, Abbas H, Ahearn T, et al. Myocardial and systemic inflammation in acute stress-induced (takotsubo) cardiomyopathy. Circulation 2019; 139:1581-1592.

18. Templin C, Hanggi J, Klein C, et al. Altered limbic and autonomic processing supports brain-heart axis in takotsubo syndrome. Eur Heart 2019;40:1183-1187.

19. Boeddinghaus J, Nestelberger T, Twerenbold R, et al.; APACE, BACC, and TRAPID-AMI Investigators. Impact of age on the performance of the ESC O/Ih-algorithms for early diagnosis of myocardial infarction. Eur Heart J 2018;39:3780-3794.
20. Twerenbold R, Costabel JP, Nestelberger T, et al. Outcome of applying the ESC 0/I-hour algorithm in patients with suspected myocardial infarction. J Am Coll Cardiol 2019;74:483-494.

21. Januzzi JLJr, Mahler SA, Christenson RH, et al. Recommendations for institutions transitioning to high-sensitivity troponin testing: JACC Scientific Expert Panel. J Am Coll Cardiol 2019;73:1059-1077.

22. Gibson CM, Holmes D, Mikdadi G, et al. Implantable cardiac alert system for early recognition of ST-segment elevation myocardial infarction. J Am Coll Cardiol 2019;73:1919-1927.

23. Mani P, Puri R, Schwartz GG, et al. Association of initial and serial C-reactive protein levels with adverse cardiovascular events and death after acute coronary syndrome: A secondary analysis of the VISTA-16 trial. JAMA Cardiol 2019:4:314-320.

24. Asleh R, Enriquez-Sarano M, Jaffe AS, et al. Galectin-3 levels and outcomes after myocardial infarction: A population-based study. J Am Coll Cardiol 2019;73:2286-2295.

25. Farag M, Spinthakis N, Gue YX, et al. Impaired endogenous fibrinolysis in ST-segment elevation myocardial infarction patients undergoing primary percutaneous coronary intervention is a predictor of recurrent cardiovascular events: The RISK PPCI study. Eur Heart J 2019;40:295-305.

26. Li XS, Obeid S, Wang Z, et al. Trimethyllysine, a trimethylamine $\mathrm{N}$-oxide precursor, provides near- and long-term prognostic value in patients presenting with acute coronary syndromes. Eur Heart J 2019;40:2700-2709.

27. Guerin M, Silvain J, Gall J, et al. Association of serum cholesterol efflux capacity with mortality in patients with ST-segment elevation myocardial infarction. J Am Coll Cardiol 2018;72:3259-3269.

28. Lindholm D, James SK, Gabrysch K, et al. Association of multiple biomarkers with risk of all-cause and cause-specific mortality after acute coronary syndromes: A secondary analysis of the PLATO biomarker study. JAMA Cardiol 2018;3:1 160-1 166.

29. Androulakis AFA, Zeppenfeld K, Paiman EHM, et al. Entropy as a novel measure of myocardial tissue heterogeneity for prediction of ventricular arrhythmias and mortality in post-infarct patients. JACC Clin Electrophysiol 2019;5:480-489.

30. Schupke S, Neumann FJ, Menichelli M, et al.; ISAR-REACT 5 Trial Investigators. Ticagrelor or prasugrel in patients with acute coronary syndromes. N Engl J Med 2019;381:1524-I534.

31. Redfors B, Dworeck C, Haraldsson I, et al. Pretreatment with P2YI2 receptor antagonists in ST-elevation myocardial infarction: a report from the Swedish Coronary Angiography and Angioplasty Registry. Eur Heart J 2019;40:1202-1210.

32. Ibanez B, James S, Agewall S, et al.; ESC Scientific Document Group. 2017 ESC Guidelines for the management of acute myocardial infarction in patients presenting with ST-segment elevation: The Task Force for the management of acute myocardial infarction in patients presenting with STsegment elevation of the European Society of Cardiology (ESC). Eur Heart J 2018;39:119-177.

33. Abtan J, Steg PG. Pre-treatment with a P2YI2 antagonist before $\mathrm{PCl}$ in STEMI: Why should we wait? Eur Heart J 2019;40:121 1-1213.

34. Lemkes JS, Janssens GN, van der Hoeven NW, et al. Timing of revascularisation in patients with transient ST-segment elevation myocardial infarction: A randomised clinical trial. Eur Heart J 2019;40:283-291.

35. Mehta SR, Wood DA, Storey RF, et al.; COMPLETE Trial Steering Committee and Investigators. Complete revascularisation with multivessel $\mathrm{PCl}$ for myocardial infarction. N Engl J Med 2019;38 |:|4| | - |421.

36. Stone GW, Genereux P, Harrington RA, et al. Impact of lesion complexity on peri-procedural adverse events and the benefit of potent intravenous platelet adenosine diphosphate receptor inhibition after percutaneous coronary intervention: Core laboratory analysis from 10854 patients from the CHAMPION PHOENIX trial. Eur Heart I 20 I 8;39:4 I I 2-4 I 2 I. 


\section{REFERENCES}

37. Fujino A, Kadohira $T$, Redfors B, et al. Significant association among residual SYNTAX score, non-culprit major adverse cardiac events, and greyscale and virtual histology intravascular ultrasound findings: A substudy from the PROSPECT study. Eurolntervention 2019;14:1676-1684.

38. Johnson TW, Raber L, di Mario C, et al. Clinical use of intracoronary imaging. Part 2: Acute coronary syndromes, ambiguous coronary angiography findings, and guiding interventional decision-making: an expert consensus document of the European Association of Percutaneous Cardiovascular Interventions. Eur Heart J 2019;40:2566-2584

39. Aradi D, Gross L, Trenk D, et al. Platelet reactivity and clinical outcomes in acute coronary syndrome patients treated with prasugrel and clopidogrel: Apre-specified exploratory analysis from the TROPICAL-ACS trial. Eur Heart J 2019;40:1942-1951.

40. De Maria GL, Alkhalil M, Wolfrum M, et al. Index of microcirculatory resistance as a tool to characterise microvascular obstruction and to predict infarct size regression in patients with STEMI undergoing primary PCI. JACC Cardiovasc Imaging 2019;1 2:837-848.

41. Nepper-Christensen L, Lønborg J, Høfsten DE, et al. Impact of diagnostic ECG-to-wire delay in STEMI patients treated with primary PCI: A DANAMI-3 substudy. Eurolntervention 2018;14:700-707.

42. De Maria GL, Cuculi F, Patel N, et al. How does coronary stent implantation impact on the status of the microcirculation during primary percutaneous coronary intervention in patients with ST-elevation myocardial infarction? Eur Heart J 2015;36:3165-3177.

43. Morales-Ponce FJ, Lozano-Cid FJ, Martinez-Romero P, et al. Intracoronary tenecteplase versus abciximab as adjunctive treatment during primary percutaneous coronary intervention in patients with anterior myocardial infarction. Eurolntervention 2019;14:1668-1675.

44. McCartney PJ, Eteiba H, Maznyczka AM, et al.; T-TIME Group. Effect of lowdose intracoronary alteplase during primary percutaneous coronary intervention on microvascular obstruction in patients with acute myocardial infarction: A randomised clinical trial. JAMA 2019;321:56-68.

45. Mathias WJr, Tsutsui JM, Tavares BG, et al.; MRUSMI Investigators Sonothrombolysis in ST-segment elevation myocardial infarction treated with primary percutaneous coronary intervention. I Am Coll Cardiol 2019;73:2832-2842

46. Hausenloy DJ, Kharbanda RK, Moller UK, et al.; CONDI-2/ERIC-PPC Investigators. Effect of remote ischaemic conditioning on clinical outcomes in patients with acute myocardial infarction (CONDI-2/ERIC-PPCl): A singleblind randomised controlled trial. Lancet 2019;394:1415-1424.

47. Hall M, Bebb OJ, Dondo TB, e al. Guideline-indicated treatments and diagnostics, GRACE risk score, and survival for non-ST elevation myocardia infarction. Eur Heart J 2018;39:3798-3806.

48. Szummer K, Wallentin L, Lindhagen L, et al. Relations between implementation of new treatments and improved outcomes in patients with non-STelevation myocardial infarction during the last 20 years: Experiences from SWEDEHEART registry 1995 to 2014. Eur Heart J 20I 8;39:3766-3776.

49. Doimo S, Fabris E, Piepoli M, et al. Impact of ambulatory cardiac rehabilitation on cardiovascular outcomes: A long-term follow-up study. Eur Heart 2019:40:678-685

50. Jones DA, Rathod KS, Koganti S, et al. The association between the public reporting of individual operator outcomes with patient profiles, procedural management, and mortality after percutaneous coronary intervention: An observational study from the Pan-London PCl (BCIS) Registry using an interrupted time series analysis. Eur Heart J 2019;40:2620-2629.

51. Berg DD, Wiviott SD, Braunwald E, et al. Modes and timing of death in 66252 patients with non-ST-segment elevation acute coronary syndromes enrolled in I4 TIMI trials. Eur Heart I 20 18;39:38 I0-3820.

52. Bradley SM, Borgerding JA, Wood GB, et al. Incidence, risk factors, and outcomes associated with in-hospital acute myocardial infarction. JAMA Netw Open 2019;2:el 87348.
53. Saw J, Starovoytov A, Humphries K, et al. Canadian spontaneous coronary artery dissection cohort study: In-hospital and 30-day outcomes. Eur Heart J 2019;40:1 188-1197.

54. Gili S, Cammann VL, Schlossbauer SA, et al. Cardiac arrest in takotsubo syndrome: results from the InterTAK Registry. EurHeart J 2019;40: 2|42-215|.

55. Cammann VL, Sarcon A, Ding KJ, et al. Clinical features and outcomes of patients with malignancy and takotsubo syndrome: Observations from the International Takotsubo Registry. J Am Heart Assoc 2019;8:e0 I 088 I.

56. Dreyer RP, Tavella R, Curtis JP, et al. Myocardial infarction with nonobstructive coronary arteries as compared with myocardial infarction and obstructive coronary disease: Outcomes in a Medicare population. Eur Heart J 2019; doi: 10.1093/eurheartj/ehz403.

57. Stehli J, Martin C, Brennan A, et al. Sex differences persist in time to presentation, revascularisation, and mortality in myocardial infarction treated with percutaneous coronary intervention. J Am Heart Assoc 20 1 9;8:e0 2161 .

58. Meyer MR, Bernheim AM, Kurz DJ, et al. Gender differences in patient and system delay for primary percutaneous coronary intervention: Current trends in a Swiss ST-segment elevation myocardial infarction population. Eur Heart I Acute Cardiovasc Care 2019:8:283-290.

59. Hao Y, Liu J, Liu J, et al. Sex differences in in-hospital management and outcomes of patients with acute coronary syndrome. Circulation 2019, 139:1776-1785.

60. Vicent L, Ariza-Solé A, Alegre $O$, et al. Octogenarian women with acute coronary syndrome present frailty and readmissions more frequently than men. Eur Heart J Acute Cardiovasc Care 2019;8:252-263.

61. Patel N, Gupta A, Doshi R, et al. In-hospital management and outcomes after ST-segment-elevation myocardial infarction in Medicaid beneficiaries compared with privately insured individuals. Circ Cardiovasc Qual Outcomes 2019;| 2:e00497|.

62. Graham GN, Jones PG, Chan PS, et al. Racial disparities in patien characteristics and survival after acute myocardial infarction. JAMA Netw Open 2018; I:el 84240 